\title{
Uranium vacancy mobility at the $\Sigma 5$ symmetric tilt and $\Sigma 5$ twist grain boundaries in $\mathrm{UO}_{2}$
}

\author{
Blas Pedro Uberuaga ${ }^{\mathrm{a}}$, David A. Andersson ${ }^{\mathrm{a}}$ \\ ${ }^{a}$ Materials Science and Technology Division, Los Alamos National Laboratory, Los Alamos, NM 87545
}

\begin{abstract}
Ionic transport at grain boundaries in oxides dictates a number of important phenomena, from ionic conductivity to sintering to creep. For nuclear fuels, it also influences fission gas bubble nucleation and growth. Here, using a combination of atomistic calculations and object kinetic Monte Carlo (okMC) simulations, we examine the kinetic pathways associated with uranium vacancies at two model grain boundaries in $\mathrm{UO}_{2}$. The barriers for vacancy motion were calculated using the nudged elastic band method at all uranium sites at each grain boundary and were used as the basis of the okMC simulations. For both boundaries considered - a simple tilt and a simple twist boundary - the mobility of uranium vacancies is significantly higher than in the bulk. For the tilt boundary, there is clearly preferred migration along the tilt axis as opposed to in the perpendicular direction while, for the twist boundary, migration is essentially isotropic within the boundary plane. These results show that cation defect mobility in fluorite-structured materials is enhanced at certain types of grain boundaries and is dependent on the boundary structure with the tilt boundary exhibiting higher rates of migration than the twist boundary.
\end{abstract}

Keywords: urania, grain boundaries, cation migration, kinetic Monte Carlo

\section{Introduction}

An important consequence of the fissioning process occurring during burnup of nuclear fuels is the formation of fission products. These fission products alter the thermomechanical properties of the fuel. They also lead to macroscopic changes in the fuel structure, including the formation of bubbles that are connected to swelling of the fuel [1]. Subsequent release of fission gases increase the pressure in the plenum and can cause changes in the properties of the fuel pin itself [2]. It is thus imperative to understand how fission products, and fission gases in particular, behave within the fuel in order to predict the performance of the fuel under operating conditions. In addition to influencing fission gas bubble nucleation, cation mobility at grain boundaries also impacts creep [3] and sintering kinetics [4], particularly in nanostructured materials.

Fission gas redistribution within the fuel is governed by mass transport and the presence of sinks such as impurities, dislocations, and grain boundaries. Thus, to understand how the distribution of fission gases evolves in the fuel, we must understand the underlying transport mechanisms, tied to the concentrations and mobilities of defects within the material, and how these gases interact with microstructural features that might act as sinks. The mobility of cation defects and fission products in bulk urania $\left(\mathrm{UO}_{2}\right)$ has received significant attention in the litera- ture [5-19] as has the segregation of various fission products to microstructural features such as bubbles, dislocations and grain boundaries [20-23]. However, once a fission product has reached a sink, such as a grain boundary, its mobility may be different there than in the grain interior and predicting how, for example, bubbles nucleate within grain boundaries necessitates an understanding of how fission gases diffuse within boundaries. That is the goal of the present work.

In this manuscript, we describe atomic level simulations of uranium vacancy diffusion in the presence of a $\Sigma 5$ symmetric tilt and a $\Sigma 5$ twist boundary in $\mathrm{UO}_{2}$. These boundary were chosen as they are the simple grain boundaries that are amenable to study with atomistic methods. Further, we considered these boundaries in previous work on segregation and they serve as a starting point for understanding defect mobility at boundaries more generally. We use a combination of molecular statics calculations and kinetic Monte Carlo (kMC) to determine how the mobility of uranium vacancies is altered at these particular grain boundaries as compared to within bulk $\mathrm{UO}_{2}$. Given that the diffusion of fission gases such as Xe are tied to the mobility of uranium vacancies, these results provide insight into how fission gas mobility differs at grain boundaries compared to bulk urania.

Email address: blas@lanl.gov (Blas Pedro Uberuaga) 


\section{Methodology}

\subsection{Potential}

We use the U-O potential derived by Basak et al. [24] for these calculations. It should be noted that there are a number of potentials that have been developed for $\mathrm{UO}_{2}$ [25] Our choice of the Basak potential is based on our previous experience that it agrees reasonably well with density functional theory (DFT) calculations of grain boundary (GB) structure [26]. Furthermore, it provides a good description of the elastic constants of $\mathrm{UO}_{2}$ leading to a more accurate representation of the strain energy [25]. However, all of the $\mathrm{UO}_{2}$ potentials are limited in their applicability, and none of them can accurately describe any arbitrary configuration within $\mathrm{UO}_{2}$. We expect that the qualitative trends we describe here are independent of the potential, though the details may differ. We also use the Basak potential for all calculations for convenience in comparing results with previous work on segregation [22].

\subsection{Molecular statics}

\subsection{1. $\Sigma 5$ tilt}

Our approach involves two steps. First, we characterize all of the diffusion pathways (within certain assumptions) for uranium vacancies as a function of position of the vacancy near the grain boundary plane. This is done by first creating a vacancy at every possible site within a simulation cell that contains two $\Sigma 5$ tilt grain boundaries (see Figure 1) and minimizing the structure. Then, for each of these sites, the neighboring uranium atoms are displaced into the vacancy such that the vacancy moves one nearest neighbor distance in all possible directions. We determine the possible directions by finding all uranium neighbors within a distance of $0.4 \mathrm{~nm}$ from the vacant site. In the bulk, this results in 12 nearest neighbor uranium ions, but near the grain boundary, there are some sites that have 11, 12 , or 13 neighbors. These final positions of the vacancy are then minimized. At this point, we have the initial and final positions for the vacancy to hop from each possible site in the system to each possible neighbor. These positions are then used as the starting point for nudged elastic band (NEB) [27] calculations to determine the saddle point for that particular hop of the vacancy. Given that in our simulation cell for the $\Sigma 5$ tilt grain boundary there are 480 possible vacancy sites and roughly 12 neighbors per site, this results in 5760 calculations. These calculations give the energy barrier $E_{m}$ for the vacancy to hop via any of these events.

Clearly, not all of these events are independent, as a vacancy hop from site $i$ to neighbor $j$ is symmetrically opposite of that from site $j$ to neighbor $i$. However, by performing this brute force calculation, we have redundant information to ensure the calculations converged correctly. In fact, for some environments these minimizations proved to be very sensitive to small perturbations in the local structure as minimizing symmetrically equivalent vacancy structures resulted in different energies. (Even calculating the migration barrier for a uranium vacancy in bulk urania can be problematic because of the rather complex nature of the pathway [18].) Sometimes this was because the minimizer stopped before it was fully minimized (it artificially reach the convergence criterion too early) or because different local arrangements of oxygen resulted in different minima. To ensure that our database of sites and events connecting sites was consistent, whenever we have different energetics for symmetrically equivalent structures, we chose the lowest one for all symmetrically equivalent sites and set the barrier between them at the lowest energy converged NEB value for that set of minima. If the saddle reported by the NEB calculation was smaller than either of the two end points (which essentially means that one of the end points was the highest energy image along the NEB), we set it equal to the higher of the two end points. These assumptions might result in some quantitative inaccuracies as the correct path might still not be identified from this procedure, but we expect it will have little effect on the physical trends we report below.

Figure 2 shows the relative formation energy of vacancies near the $\Sigma 5$ tilt boundary. As is clearly evident, the vacancy is strongly attracted to the grain boundary plane. Figure 3 shows the relative saddle energies as a function of the energy difference between the final and initial minima $(\Delta E)$. We see that there are large variations of the saddle energy for any given value of $\Delta E$; that is, there is no simple relationship between the two (contrary to many assumptions in kinetic Monte Carlo simulations). Even when $\Delta E 0-$ when there is little difference in the vacancy energy before and after the hop - the barriers for those hops vary by about $6 \mathrm{eV}$. This shows that the kinetic behavior near the boundary will be a relatively complex competition between a number of different processes.

\subsection{2. $\Sigma 5$ twist}

For the $\Sigma 5$ twist grain boundary, we did not calculate the migration barrier for all possible uranium vacancy sites. Rather, we focused on the four atomic planes nearest the grain boundary plane. Given the size of our supercell, this means we considered 80 different sites. When the number of neighbors for each site is considered, the total number of migration pathways calculated were 928, considerably less than performed for the $\Sigma 5$ tilt GB. The rationale for limiting our consideration to sites near the GB plane is a consequence of the fact that the vacancy is typically strongly attracted to the GB and thus does not perform excursions far from the GB plane. Thus, only those sites near the GB are important on the time scales of the simulations discussed here and, thus, for computational efficiency, we limited the calculations for this boundary to a narrow region near the boundary plane. The structure of the $\Sigma 5$ twist boundary is shown in Fig. 4; the ions highlighted in pink in the side view are those that were considered in these calculations.

The formation energy of the vacancy as a function of 

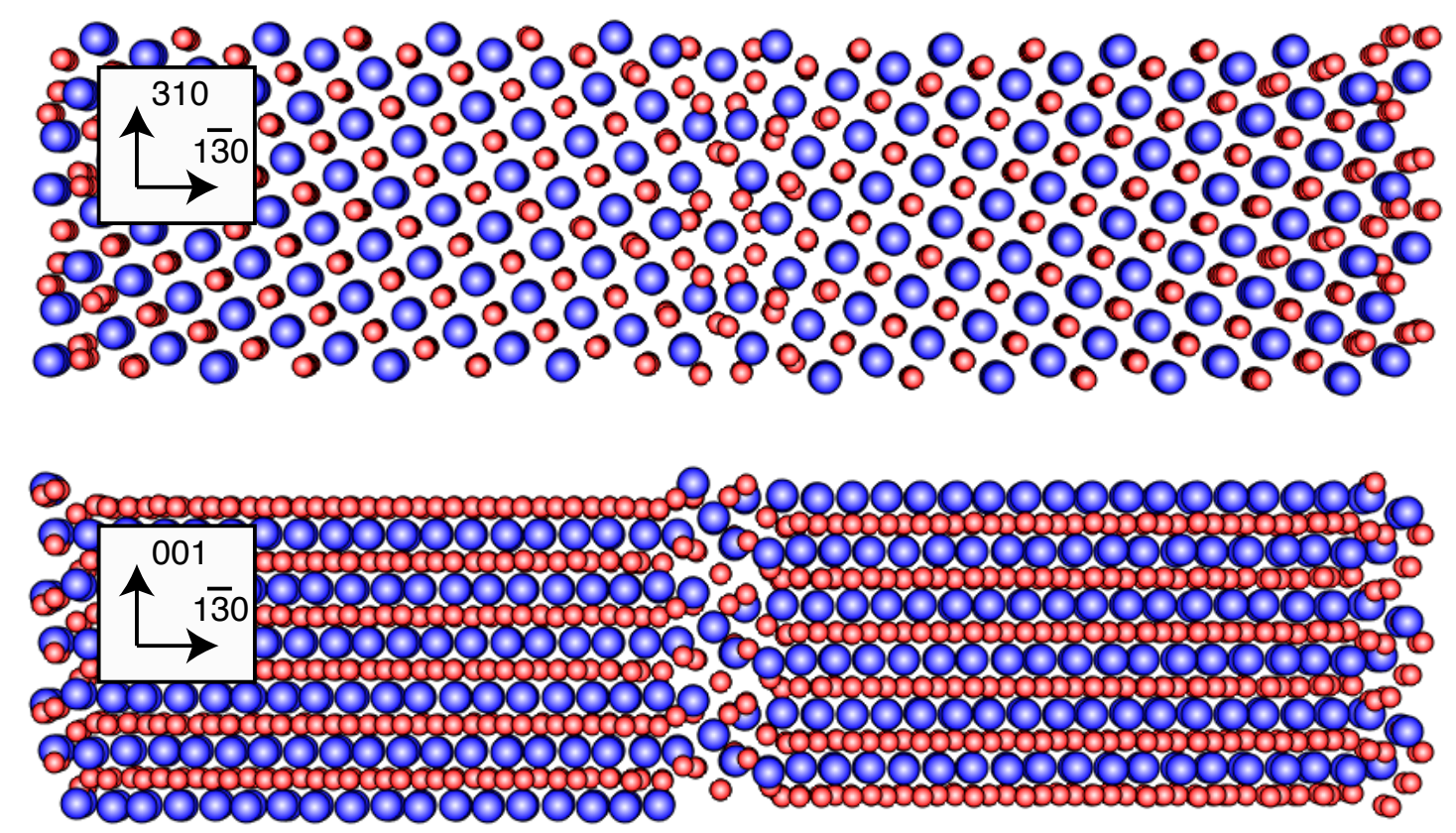

Figure 1: Structure of the $\Sigma 5$ symmetric tilt grain boundary in $\mathrm{UO}_{2}$. Top, the view down the tilt axis. Bottom, the view perpendicular to the tilt axis. The blue spheres indicate uranium ions, the red spheres are oxygen ions. There are two grain boundaries in the simulation cell, one in the center and the other at the periodic boundary of the cell. The orientation of the left-most grain is indicated.

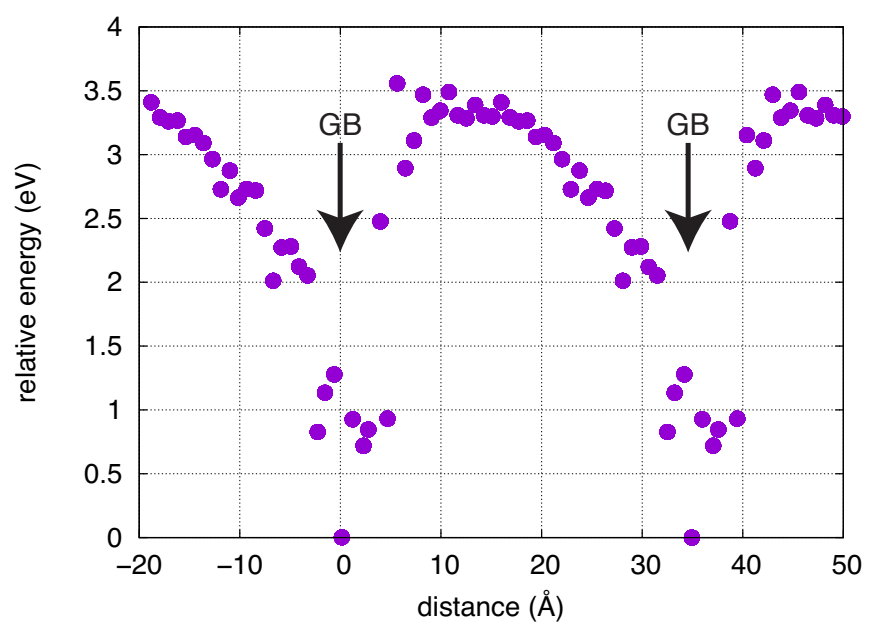

Figure 2: Relative formation energy of the uranium vacancy as a function of position from the two grain boundary planes for the $\Sigma 5$ tilt GB. The position of the two grain boundary planes are indicated by the arrows. The energies are relative to the lowest energy site for the vacancy within the system. Distance is measured from the left-most grain boundary plane.

position near the grain boundary plane of the $\Sigma 5$ tilt GB is shown in Fig. 5 while the relative saddle energies are shown in Fig. 6. The primary difference between this GB and the $\Sigma 5$ tilt shown in Figs. 2 and 3 is in the energetics of the vacancy itself. While in the case of the $\Sigma 5$ tilt there are deep trap states at the GB plane, in the case of the $\Sigma 5$ twist, the situation is a bit more complex. There are trap states, with an estimated trapping energy of about

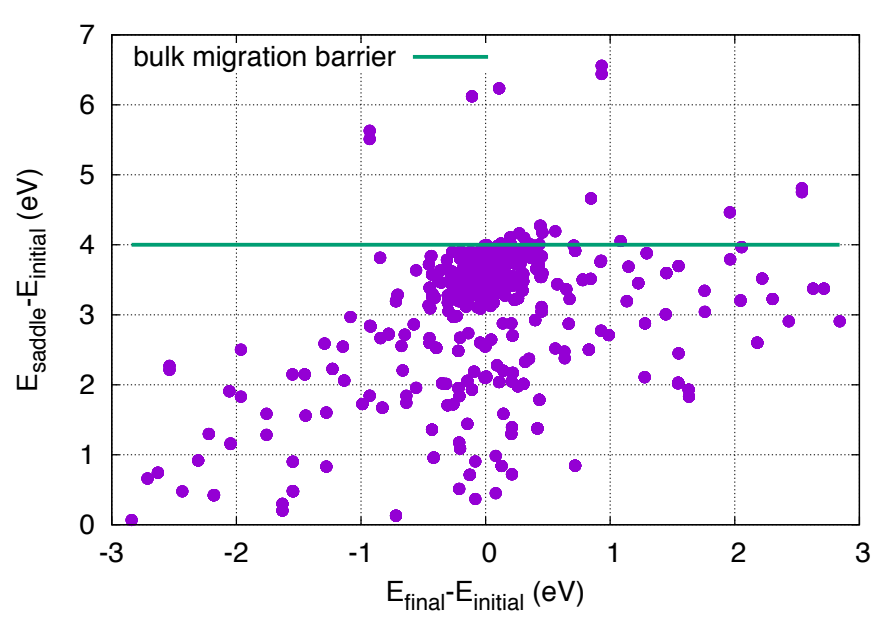

Figure 3: Relative saddle energies as a function of the difference in energy between the final and initial state of the event for the $\Sigma 5$ tilt GB. While there is a rough correlation, it is certainly not linear, as often assumed. For any given difference in energy, there are a range of saddle energies, up to $6 \mathrm{eV}$ in some cases. The migration energy of the uranium vacancy in bulk $\mathrm{UO}_{2}$ is provided as reference.

$1 \mathrm{eV}$ (also compare to the behavior of Xe at these GBs, which essentially tracks the behavior of $U$ vacancies [22]). However, there are also sites at the GB plane where the vacancy is very unfavorable. Thus, diffusion within the GB plane will be a complicated path through the plane itself with the vacancy avoiding certain sites and stuck for long times in others. The relative barriers themselves (Fig. 6) show overall trends not very different from the $\Sigma 5$ tilt GB. 

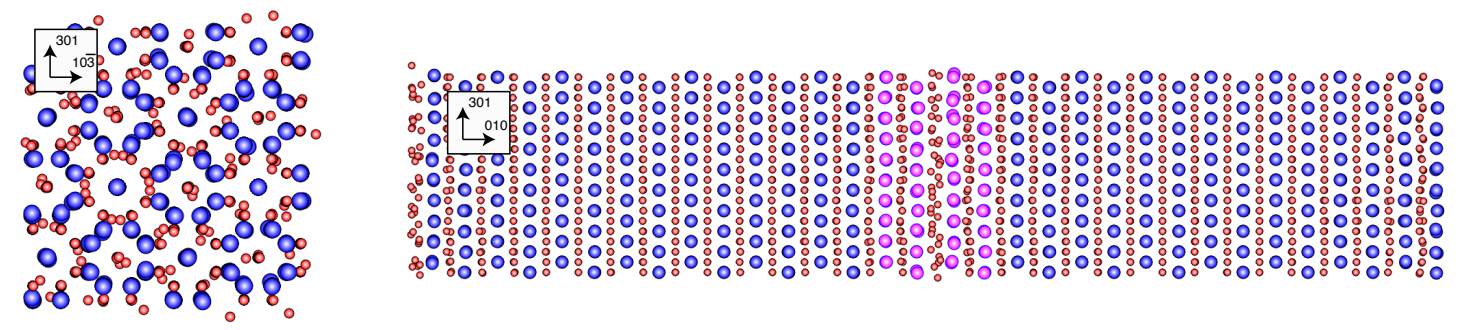

Figure 4: Structure of the $\Sigma 5$ twist grain boundary in $\mathrm{UO}_{2}$. Left, the view along the GB normal direction. Right, the view perpendicular to the GB normal. The blue spheres indicate uranium ions, the red spheres are oxygen ions. There are two grain boundaries in the simulation cell, one in the center and the other at the periodic boundary of the cell. The purple ions in the right panel highlight the sites for which migration energies are calculated and used in the subsequent KMC simulations. The orientation of the upper grain (left panel) and the left-most grain (right panel) is indicated.

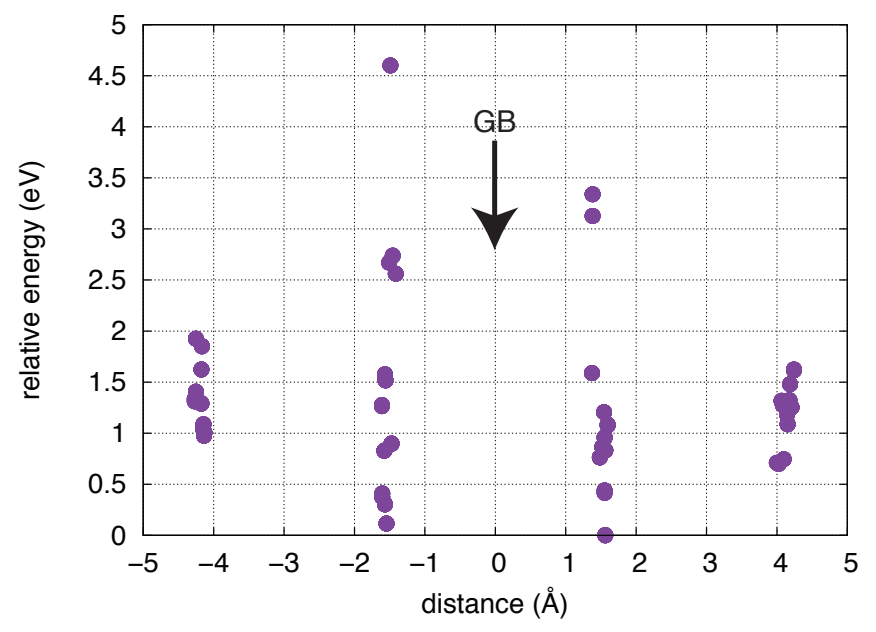

Figure 5: Relative formation energy of the uranium vacancy as a function of site near the $\Sigma 5$ twist grain boundary plane. Only those sites considered in the KMC simulations are shown in this plot. Distance is measured from the mirror plane of the grain boundary.

\subsection{Kinetic Monte Carlo}

The results from atomistic calculations are used as the input for object kinetic Monte Carlo (okMC) [28] simulations in which the vacancy is allowed to hop from uranium lattice site to uranium lattice site. While the lattice is assumed rigid, its initial structure is identical to that of the starting boundary structures as described above (see Figs. 1 and 4). okMC is a stochastic algorithm that is in principle exact if all possible kinetic pathways and their rates are included in the simulation. We have not included every possible event in our rate catalog, so the trajectories predicted here would not be identical to a corresponding molecular dynamics (MD) trajectory. However, we expect the basic physical trends to be similar. Further, the time scales necessary to simulate uranium vacancy diffusion, especially at lower temperatures, would not be possible with MD.

The rate $k_{i j}$ of a given process taking the vacancy from site $i$ to $j$ is given by $k_{i j}=\nu_{i j} \exp \left(-E_{m}^{i j} / k_{b} T\right)$ where $k_{B}$ is the Boltzmann constant and $T$ is temperature. As stated above, $E_{m}^{i j}$ is directly obtained from the atomistic calcu-

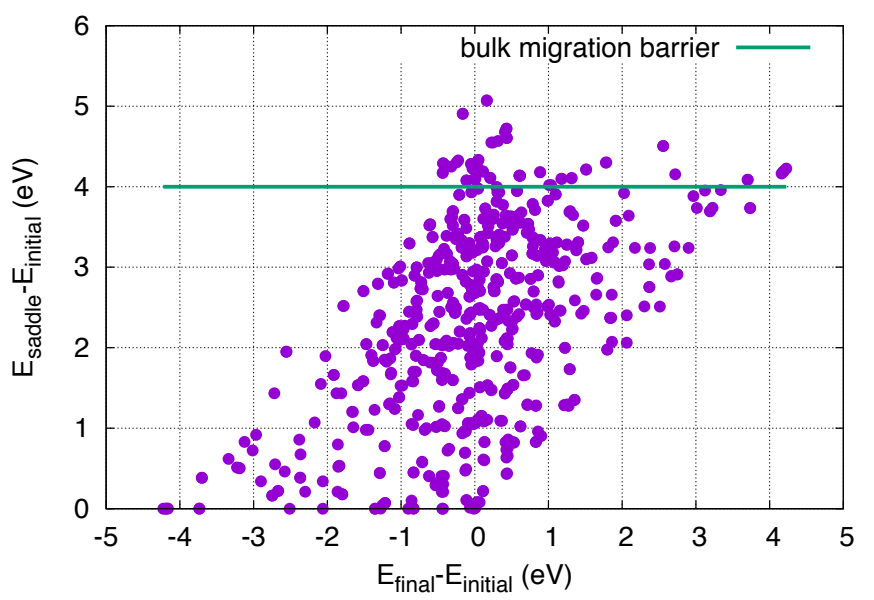

Figure 6: Relative saddle energies as a function of the difference in energy between the final and initial state of the event for the $\Sigma 5$ twist GB. While there is a rough correlation, it is certainly not linear, as often assumed. For any given difference in energy, there are a range of saddle energies, up to $6 \mathrm{eV}$ in some cases.

lations. In principle, $\nu$ could so be derived as well, but calculating $\nu_{i j}$ is a computationally expensive task and is a relatively minor contributor to differences in $k_{i j}$ for different processes, which is typically dominated by $E_{m}^{i j}$ (residing within the exponential). Thus, here we assume $\nu_{i j}=10^{13} / \mathrm{s}$ for all $i$ and $j$. This is close to the Debye frequency for $\mathrm{UO}_{2}, 1.6 \times 10^{13} / \mathrm{s}[29]$, though the actual value will only modify the prefactor for diffusion obtained from the okMC simulations, not the activation energy. The bigger assumption here is that the prefactor is constant for all processes, which is clearly not strictly true. However, the computational cost in computing all relevant prefactors is prohibitive and, as mentioned, would likely only slightly influence the final behavior of the vacancy, and so that assumption is made here.

Given the rate catalog defined by $k_{i j}$, an okMC simulation proceeds as follows (see Ref. [28] for details). For the current position $i$ of the vacancy, all of the possible hops are identified, and the total rate $k_{i}^{\text {total }}=\sum_{j=1}^{N} k_{i j}$ where $N$ is the total number of neighboring positions for 
this vacancy. One of these events is then chosen at random, weighted by its relative rate: $k_{i j} / k_{i}^{\text {total }}$. The time for the event is correspondingly calculated via the equation $\Delta t=\ln (1 / s) / k_{i}^{\text {total }}$, where $s$ is a random number between 0 and 1 , and added to the total simulation time. The vacancy is moved into the chosen neighboring site and the steps repeated. The simulations reported here are run for 5 million okMC steps.

By monitoring the mean square displacement (MSD) during the course of the simulation, we can measure the diffusivity of the defect: $\left\langle r^{2}\right\rangle \sim D t$, where $r$ is the MSD of the particle or collection of particles in the simulation over a time $t$. The $D$ that results from this equation is not exactly the diffusion constant as measured by experiment as we have a constant defect concentration in our simulations while the defect concentration in experiment varies with temperature. Thus, we are extracting the mobility per defect of the uranium vacancy. In the simulations reported here, we have only one vacancy within the simulation cell.

In the measurements of defect diffusion reported here, we averaged over 10 simulations at each temperature considered. For each simulation, there are error bars for the extracted diffusivity $D$; here, those are ignored. Rather, we report error bars related to the standard error associated with the $D$ averaged over the 10 simulations. The error bars (computed as the standard error) range from $0.1 \%$ at the high temperatures to about $8 \%$ at the lowest temperature.

As the $\Sigma 5$ tilt grain boundary is highly anisotropic, with faster diffusion along the tilt axis than perpendicular to it, the mobilities along the tilt axis (along $x$ in our coordinate system) will be very different than along $y$, the direction perpendicular to the tilt axis. We thus measure mobilities both along $x$ and along $y$ to determine the relative mobilities, or anisotropies in diffusion, in the two dimensions. In the case of the $\Sigma 5$ twist boundary, the two in-plane directions are equivalent and no difference in diffusion along the two directions is expected. For consistency, the anisotropy in this boundary is also measured.

We note that this study would have been extremely challenging with conventional molecular dynamics (MD). An MD simulation at $2000 \mathrm{~K}$ for $1 \mathrm{~ns}$ at the $\Sigma 5$ tilt GB lead to only about 5 events involving the uranium vacancy (after it was placed at the GB plane), none of which lead to net migration of the vacancy. The temperature cannot be raised much higher as the oxygen sublattice will premelt (at a temperature between 2300-2700 K [24]). Thus, the oKMC approach is probably the most efficient to provide long-time trajectories for uranium vacancy migration suitable for extracting migration properties.

\section{Results}

\section{1. $\Sigma 5$ tilt}

Figure 7 shows example trajectories at four different temperatures. As is clearly evident, as the temperature is increased from $2500 \mathrm{~K}$ to $10,000 \mathrm{~K}$, the diffusive behavior of the vacancy changes from $1 \mathrm{D}$ to $2 \mathrm{D}$ within the grain boundary plane. The total time for these simulations ranges from 0.5 to $5 \mu \mathrm{s}$. This is not such a great difference, considering the wide range of temperatures, but this is because the rate for the fastest processes is very similar, a consequence of the low barriers associated with them.

A note about the temperatures is in order. Clearly, these are physically meaningless temperatures, as urania melts at temperatures just above $3000 \mathrm{~K}$ [30]. However, even with the advantages provided by okMC, we are unable to simulate long enough times at operating temperatures to see diffusive behavior. That is, to observe net diffusion at temperatures of $1000 \mathrm{~K}$ or below would require many billions (if not more) of okMC steps. Thus, we raise the temperature to artificially high values to extract trends in diffusivity as a function of temperature and then extrapolate that behavior to lower temperatures of interest.

Given trajectories such as those illustrated in Fig. 7, we then measure $D$ as described above. The results are presented in Fig. 8 for diffusion both parallel and perpendicular to the tilt axis. Even at the artificially high temperature of $10,000 \mathrm{~K}$, there is still significant differences in the mobility of the vacancy along $x$ compared to along $y$. This difference only increases with decreasing temperature. The ratio of $D$ along vs normal to the tilt axis is shown in Fig. 9. This anisotropy ratio increases from a factor of about 2 at $10,000 \mathrm{~K}$ to a factor of about 400 at $2500 \mathrm{~K}$. If we extrapolate to $1000 \mathrm{~K}$, the relative anisotropy factor would increase to nearly $10^{7}$ (based on a linear extrapolation of the data in Fig. 9).

We can understand this behavior by examining the topology of saddles that connect the various sites at the grain boundary plane, as shown in Fig. 10. We see that, along the tilt axis, there is a set of low energy sites (black circles) that are connected by relatively lower barriers (smaller than $1 \mathrm{eV}$ ). This pathway does not center on the site at the mirror plane of the grain boundary, but on the $U$ sites next to it. Further, the pathway jumps between equivalent sites on either side of the mirror plane, as illustrated in the lower panel of Fig. 10. No such low-energy pathway exists along $y$, perpendicular to the tilt axis. The smallest barrier that takes the vacancy all the way along the $y$ dimension to a new equivalent position and could describe net transport is roughly $2 \mathrm{eV}$ or so.

The effective migration energies of vacancies can be extracted from the diffusivities presented in Fig 8 by fitting the results to an Arrhenius expression

$$
D=D_{0} \exp \left(-E_{m} / k_{B} T\right)
$$

Doing so, we find effective migration energies of $E_{m}^{\|} 0.8$ $\mathrm{eV}$ and $E_{m}^{\perp} 2.1 \mathrm{eV}$, consistent with the rough estimate made by looking at the saddle landscape in Fig. 10. It is interesting that both of these migration energies are significantly lower than the bulk migration energy predicted by this potential of $4 \mathrm{eV}$. At any temperature at which bulk 

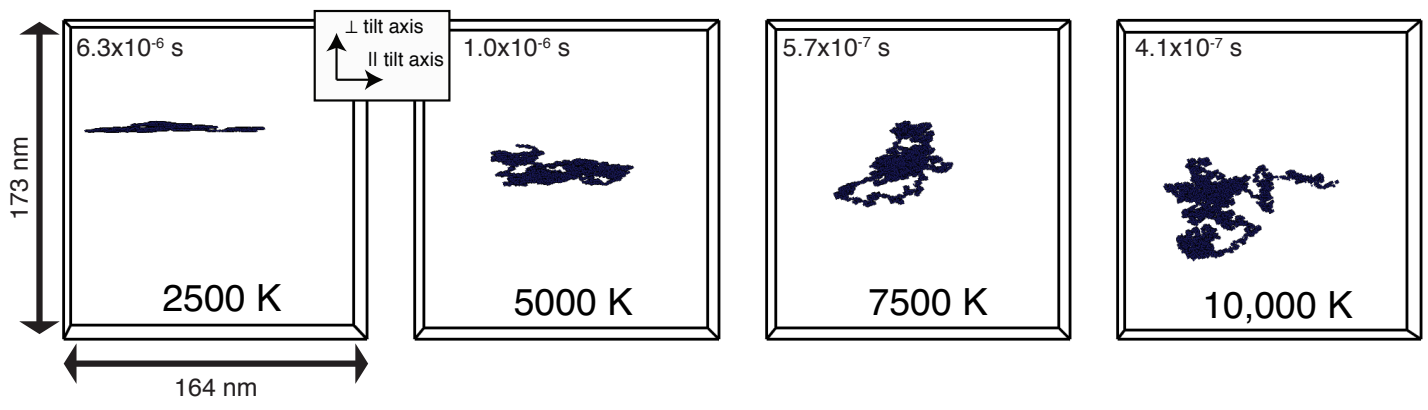

Figure 7: Example trajectories at each temperature considered $(2500 \mathrm{~K}, 5000 \mathrm{~K}, 7500 \mathrm{~K}$, and $10,000 \mathrm{~K})$ for a vacancy diffusing at the $\Sigma 5$ tilt GB. There is a clear transition from one-dimensional to two-dimensional motion as the temperature is increased. The direction along the tilt axis is indicated for clarity. The time scale for each simulation is also indicated in the upper left corner of each frame.

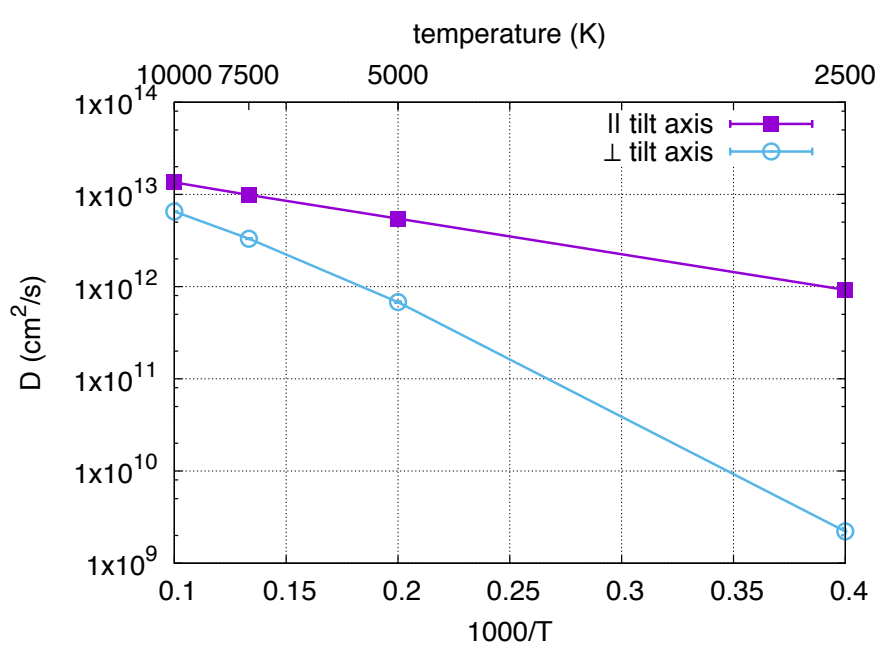

Figure 8: Diffusivity of the uranium vacancy within the grain boundary plane at the $\Sigma 5$ tilt GB, both in along the tilt axis and perpendicular to it. Diffusivity is much higher along the tilt axis than perpendicular to it. Error bars are smaller than or on the order of the size of the symbols in the figure.

migration is active, diffusion in both $x$ and $y$ will be active. However, diffusion along the tilt axis will be so much faster that the vacancy will likely reach any strong sink (triple junction or line junction between grains, surfaces, or bubbles) via this $1 \mathrm{D}$ mechanism long before diffusion perpendicular to the tilt axis occurs.

Another feature that can be observed in Fig. 8 is that, while the diffusivity along the tilt axis is Arrhenius over the temperature range considered, the same is not true for migration perpendicular to the tilt axis. There is a clear bend in the curve. Often, such a trend indicates anharmonic effects in the migration barrier, but that is explicitly not possible in these simulations as every rate is treated, by definition, as harmonic. Thus, this is an indicator that as the temperature varies, different barriers become important. This must be due to a multiplicity effect for higher barriers: while they are of higher energy, there are more of them, so they can start to be more important at high temperatures than the lower barriers.

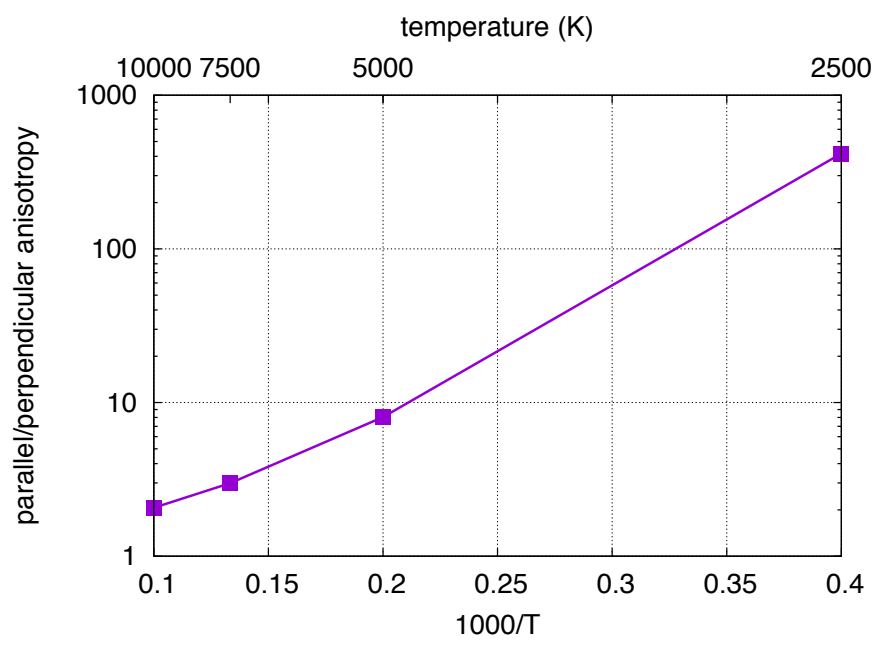

Figure 9: Anisotropy in diffusivities parallel vs perpendicular to the GB tilt axis as a function of temperature at the $\Sigma 5$ tilt GB. As the temperature is decreased, the anisotropy grows very quickly (note the $\log$ scale).

\section{2. $\Sigma 5$ twist}

In the case of the $\Sigma 5$ twist GB, we can only examine a limited temperature range given the fact that we have only included a subset of all of the possible sites for the uranium vacancy within the okMC model. In particular, we find that once we get to a temperature above $5000 \mathrm{~K}$, there is significant sampling of the sites furthest from the GB plane (the first and last pink planes in Fig. 4); thus, we restrict our okMC simulations to temperatures 5000 $\mathrm{K}$ and below. It is possible that, even at lower temperatures, on much longer time scales the vacancy will escape the grain boundary and diffuse essentially as a bulk-like species. This is especially possible for this GB as the binding energy is not so large (about $1 \mathrm{eV}$ ) as compared to the $\Sigma 5$ tilt GB. That said, on the time scale of the simulations reported here, the vast majority of events kept the vacancy to the two planes on either side of the symmetry plane of the grain boundary so that, for these simulations, such excursions are not important.

Figure 11 shows representative trajectories at two different temperatures. An interesting aspect of the trajecto- 


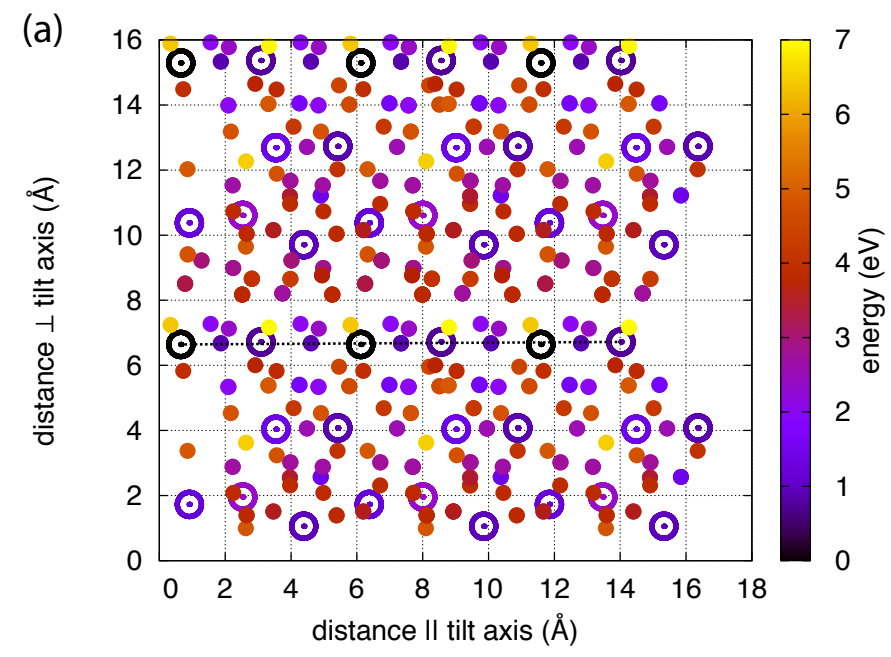

(b)

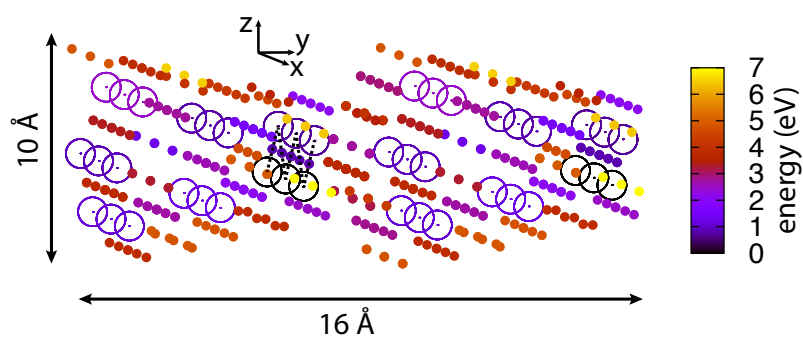

Figure 10: Schematic of the saddles connecting the various stable uranium vacancy sites at and near the grain boundary plane at the $\Sigma 5$ tilt GB. (a) View along the grain boundary normal. (b) Perspective view rotated slightly off the direction of the tilt axis $(x$ in the figure). The large open circles are stable sites (minima) while the small filled circles represent the saddles connecting them (placed exactly half way between the minima). The colors of both types of circles indicate the energy of the site or saddle. There is a low barrier path way along the tilt axis, leading to fast diffusion along that dimension. This pathway is highlighted by the dotted line. In contrast, no such pathway exists perpendicular to the tilt axis.

ries, especially apparent in the $2500 \mathrm{~K}$ simulation, is that the defect tends to move along directions that are rotated by 45 degrees to the coordinates of the simulation box (along a [102] direction in the coordinates of the upper grain). This is surprising given that the ideal structure of the boundary is perfectly symmetric with respect to the

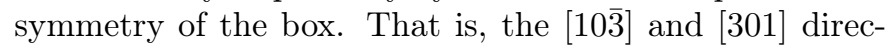
tions are equivalent in the ideal boundary. However, in the dynamics, it is apparent that the boundary is not perfectly symmetric. This becomes clearer when we examine the diffusivity of the vacancy as a function of temperature (Fig. 12). There is a small but very clear difference in the diffusivity along $[10 \overline{3}]$ as compared to along [301]. When we look closely at the structure of the boundary (Fig. 4) and the energetics of the vacancy at the boundary plane (Fig. 5), the asymmetry is obvious, particularly in the structure of the oxygen sublattice. An important, but unanswered question, is whether this asymmetry is realistic for a material such as $\mathrm{UO}_{2}$. In a simple metal, such asymmetry would not be expected. For an oxide ceramic such as $\mathrm{UO}_{2}$, the asymmetry could be a consequence of the difficulty of finding a low energy atomic structure of the grain boundary or it could be a real consequence of the complexity of the material. That is, in reality, there might be many degenerate structures associated with multiple distortions of the oxygen sublattice for a given configuration of the cation sublattice. Finally, as mentioned above, there are, at times, difficulty in finding the saddle point energy connecting sites and it is possible that the NEBs converged differently for what should be equivalent pathways, though this seems unlikely to be the sole reason for the asymmetry as the asymmetry is apparent in the structure of the GB itself.

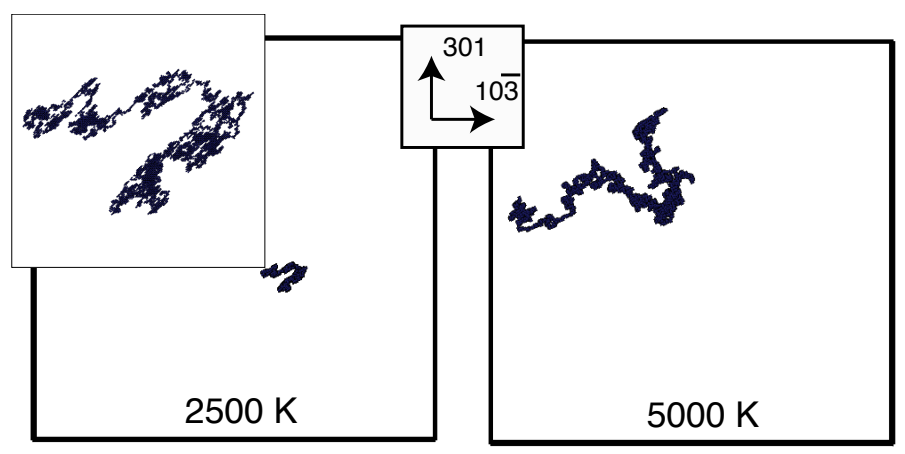

Figure 11: Example trajectories at $2500 \mathrm{~K}$ and $5000 \mathrm{~K}$ for the uranium vacancy diffusing near the $\Sigma 5$ twist GB. There is an apparent slight preference for diffusion along the $[10 \overline{3}]$ direction as opposed to the [301] direction. The inset shows a higher magnification view of the trajectory at $2500 \mathrm{~K}$.

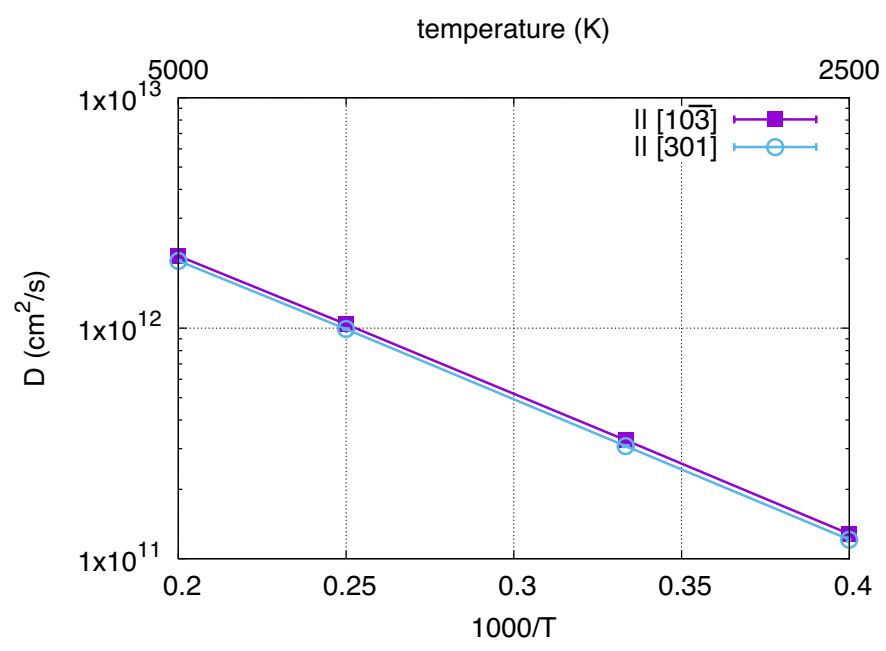

Figure 12: Diffusivity of the uranium vacancy within the grain boundary plane of the $\Sigma 5$ twist GB, both along [10̄3] and [301]. While the two diffusivities are similar, there is a clear difference that persists at all temperatures considered.

Extracting the effective migration energy for the vacancy from this okMC simulations, as we did above for the $\Sigma 5$ tilt GB, we find that the migration energy for the

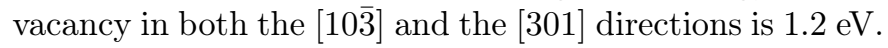
The difference in diffusivities is a consequence of slightly different prefactors for the diffusivity (hence the constant 
difference between the two diffusivities in Fig. 12). This migration energy is much lower than in the bulk $(4 \mathrm{eV})$ and lies intermediate to the two values that characterize the $\Sigma 5$ tilt GB.

\section{Discussion and Conclusions}

Previous work has found that, in some situations, defects can actually exhibit less mobility at grain boundaries than in the bulk [31-34]. This is because there are often deep trap states at grain boundaries that are not directly connected to other low energy states and thus, for diffusion, the defect has to escape the trap first. This is clearly not occurring for the two grain boundaries studied here. For the $\Sigma 5$ tilt GB, along the tilt axis, there is a string of low energy sites connected to one another via which the uranium vacancy can easily diffuse. This is consistent with results on pure tilt grain boundaries in metals [34, 35]. Even in the direction perpendicular to the tilt axis, diffusion is faster than in the bulk. For the $\Sigma 5$ twist GB, the mobility is still significantly higher than in the bulk, though a bit lower than along the tilt axis of the $\Sigma 5$ tilt GB. This is an interesting contrast with the behavior of vacancies at twist boundaries in simple metals, in which the vacancy is faster at the twist GB than at the pure tilt GBs [34]. These higher mobilities, combined with the fact that there will be a higher concentration of uranium vacancies at the grain boundary than in the bulk (due to the lower energies of formation there) means that mass transport at the $\Sigma 5$ tilt and twist grain boundaries will be significantly higher than in the bulk. Thus, in the absence of other complicating factors, once Xe makes its way to either of these boundaries, it will quickly diffuse along the grain boundary to find other sinks, such as triple junctions. The consequence is that these boundaries, at least, will not likely directly act as nucleation sites for Xe bubbles or, stated another way, nucleation of Xe bubbles at these boundaries will likely require significantly more Xe than in the bulk as the mobility is so much higher. This conclusion assumes that there are no other complicating factors that might facilitate nucleation, such as solid fission products present at the boundary. Further, it assumes that the rate of arrival of $\mathrm{Xe}$ to the boundary is much slower than the subsequent removal from the boundary via the fast diffusion. This will be true if the concentration of Xe in the bulk is small so that the Xe flux to the boundary is small. However, if the flux is high because of large Xe concentrations, the boundary will not be able to effectively remove it and the boundary could serve as a nucleation site for Xe bubbles. Finally, these two boundaries are special cases and more complex boundaries may not facilitate mass transport as efficiently as the two considered here.

Of course, the diffusion mechanism for $\mathrm{Xe}$ in $\mathrm{UO}_{2}$ is more complicated than for a single uranium vacancy, involving the motion of a second vacancy around the substitutional Xe [12]. Thus, it is possible that the topology of saddles connecting low energy sites would actually lead to significantly slower diffusion for a Xe-vacancy complex than implied by the results here for a single uranium vacancy.

To this point, we have studied only two admittedly simple grain boundaries in $\mathrm{UO}_{2}$. These represent one set of special cases for the types of GBs that might be present in $\mathrm{UO}_{2}$. To understand defect mobility at grain boundaries in $\mathrm{UO}_{2}$ more completely, a wider range of GB structures is necessary. However, finding such structures for more complex GBs is computationally and algorithmically challenging. As methods that aid in the search for low energy boundary structures mature [36], the treatment of more complicated boundary structures will become possible. However, prior simulations on self-diffusion in nanocrystalline $\mathrm{UO}_{2}$ have seen higher rates at generalized grain boundaries than in bulk [3], suggesting that, unless the boundary has a specific structure that leads to trapping sites for defects [34], mobilities will be higher at boundaries than in the bulk.

Finally, it is useful to place the resulting migration barriers found in this study in the context of temperature. For the tilt boundary, the temperature at which migration will occur along the tilt axis on the 1 second time scale, assuming a prefactor of $10^{13} / \mathrm{s}$, is about $300 \mathrm{~K}$. Migration perpendicular to the tilt axis will not become active until a temperature of about $800 \mathrm{~K}$. For the twist boundary, the associated temperature for uranium vacancy migration on the 1 second time scale is about $465 \mathrm{~K}$. In contrast, in bulk $\mathrm{UO}_{2}$, this potential predicts a migration energy of the uranium vacancy of about $4.0 \mathrm{eV}$, corresponding to a temperature of $1550 \mathrm{~K}$ for migration to become active. Thus, the grain boundaries certainly facilitate cation migration and will lead to uranium self-diffusion even at room temperature in some cases. We note that the bulk migration energy for uranium vacancies predicted by this potential $(4.0 \mathrm{eV})$ is between the values predicted by different flavors of DFT [12, 14, 16, 18], thus providing a reasonable description of the migration process of this defect.

To conclude, we have examined the mobility of the uranium vacancy, as it pertains to fission product transport, at the $\Sigma 5$ tilt and the $\Sigma 5$ twist grain boundaries. We find that the mobility of the vacancy is significantly higher at these grain boundaries than in the bulk. For the $\Sigma 5$ tilt GB, this is true for both dimensions within the grain boundary plane. However, the mobility along the tilt axis is much higher than perpendicular, meaning that effectively diffusion under operating conditions will be one-dimensional. For the $\Sigma 5$ twist GB, we also find significantly higher mobilities than in the bulk, though the mobility is slower than in the $\Sigma 5$ tilt GB. The behavior of uranium vacancies at these GBs will have ramifications for mesoscale models of fission gas evolution as the transport of fission gas along grain boundaries will depend on both the orientation of the grains and the types of grain boundaries present within the material. 


\section{Acknowledgements}

The authors thank C. R. Stanek and D. Perez for helpful discussions. This work was sponsored by the U. S. Department of Energy, Office of Nuclear Energy, Nuclear Energy Advanced Modeling and Simulation (NEAMS) program. Los Alamos National Laboratory, an affirmative action equal opportunity employer, is operated by Los Alamos National Security, LLC, for the National Nuclear Security Administration of the U.S. DOE under contract DE-AC52-06NA25396.

\section{References}

[1] J. A. Turnbull, M. O. Tucker, Swelling in $\mathrm{UO}_{2}$ under conditions of gas release, Philos. Mag. 11 (1974) 47-63.

[2] D. Olander, Nuclear fuels - present and future, J. Nucl. Mater. 389 (2009) 1-22.

[3] T. G. Desai, P. Millett, D. Wolf, Is diffusion creep the cause for the inverse hall-petch effect in nanocrystalline materials?, Mat. Sci. Eng. A 493 (2008) 41-47.

[4] H. Matzke, Ion-transport in ceramics, Philos. Mag. A 64 (1991) 1181-1200.

[5] C. R. A. Catlow, Fission-gas diffusion in uranium-dioxide, Proc. R. Soc. Lond. A 364 (1978) 473-497.

[6] R. G. J. Ball, R. W. Grimes, Diffusion of Xe in $\mathrm{UO}_{2}$, J. Chem. Soc., Faraday Trans. 86 (1990) 1257-1261.

[7] N.-D. Morelon, D. Chaleb, J.-M. Delaye, L. Van Brutzel, A new empirical potential for simulating the formation of defects and their mobility in uranium dioxide, Philos. Mag. 83 (2003) 1533-1550.

[8] Y. Yun, H. Kim, H. Kim, K. Park, Atomic diffusion mechanism of Xe in UO2, J. Nucl. Mater. 378 (1) (2008) 40-44.

[9] G. Martin, S. Maillard, L. V. Brutzel, P. Garcia, B. Dorado, C. Valot, A molecular dynamics study of radiation induced diffusion in uranium dioxide, J. Nucl. Mater. 385 (2009) 351-357.

[10] Y. Yun, O. Eriksson, P. M. Oppeneer, H. Kim, K. Park, Firstprinciples theory for helium and xenon diffusion in uranium dioxide, J. Nucl. Mater. 385 (2) (2009) 364-367.

[11] K. Govers, S. Lemehov, M. Verwerft, On the solution and migration of single $\mathrm{Xe}$ atoms in uranium dioxide: An interatomic potentials study, J. Nucl. Mater. 405 (3) (2010) 252-260.

[12] D. A. Andersson, B. P. Uberuaga, P. V. Nerikar, C. Unal, C. R. Stanek, $\mathrm{U}$ and Xe transport in $\mathrm{UO}_{2 \pm x}$ : Density functional theory calculations, Phys. Rev. B 84 (2011) 054105.

[13] X.-Y. Liu, B. P. Uberuaga, D. A. Andersson, C. R. Stanek, K. E. Sickafus, Mechanism for transient migration of xenon in $\mathrm{UO}_{2}$, Appl. Phys. Lett. 98 (2011) 151902.

[14] B. Dorado, D. A. Andersson, C. R. Stanek, M. Bertolus, B. P. Uberuaga, G. Martin, M. Freyss, P. Garcia, First-principles calculations of uranium diffusion in uranium dioxide, Phys. Rev. B 86 (2012) 035110.

[15] A. E. Thompson, C. Wolverton, Pathway and energetics of xenon migration in uranium dioxide, Phys. Rev. B 87 (2013) 104105.

[16] D. A. Andersson, P. Garcia, X.-Y. Liu, G. Pastore, M. Tonks, P. Millett, B. Dorado, D. R. Gaston, D. Andrs, R. L. Williamson, R. C. Martineau, B. P. Uberuaga, C. R. Stanek, Atomistic modeling of intrinsic and radiation-enhaned fussion gas (Xe) diffusion in $\mathrm{UO}_{2 \pm x}$ : Implications for nuclear fuel performance modeling, J. Nucl. Mater. 451 (2014) 225-242.

[17] R. Perriot, X.-Y. Liu, D. A. Andersson, C. R. Stanek, Diffusion of $\mathrm{Zr}, \mathrm{Ru}, \mathrm{Ce}, \mathrm{Y}, \mathrm{La}, \mathrm{Sr}$ and $\mathrm{Ba}$ fission products in $\mathrm{UO}_{2}$, Phys. Rev. B 91 (2015) 094103.

[18] A. Goyal, S. R. Phillpot, G. Subramanian, D. A. Andersson, C. R. Stanek, B. P. Uberuaga, Impact of homogeneous strain on uranium vacancy diffusion in uranium dioxide, Phys. Rev. B 91 (2015) 094103.
[19] M. Bertolus, M. Freyss, B. Dorado, G. Martin, K. Hoang, S. Maillard, R. Skorek, P. Garcia, C. Valot, A. Chartier, L. V. Brutzel, P. Fossati, R. W. Grimes, D. C. Parfitt, C. L. Bishop, S. T. Murphy, M. J. D. Rushton, D. Staicu, E. Yakub, S. Nichenko, M. Krack, F. Devynck, R. NgayamHappy, K. Govers, C. S. Deo, , R. K. Bahera, Linking atomic and mesoscopic scales for the modelling of the transport properties of uranium dioxide under irradiation, J. Nucl. Mater.doi:10.1016/j.jnucmat.2015.02.026.

[20] S. Hu, C. H. Henager Jr., H. L. Heinisch, M. Stan, M. I. Baskes, S. M. Valone, Phase-field modeling of gas bubbles and thermal conductivity evolution in nuclear fuels, J. Nucl. Mater. 392 (2) (2009) 292-300.

[21] A. Chartier, L. Van Brutzel, M. Freyss, Atomistic study of stability of xenon nanoclusters in uranium dioxide, Phys. Rev. B 81 (2010) 174111.

[22] P. V. Nerikar, D. C. Parfitt, L. A. Casillas Trujillo, D. A. Andersson, C. Unal, S. B. Sinnott, R. W. Grimes, B. P. Uberuaga, C. R. Stanek, Segregation of xenon to dislocations and grain boundaries in uranium dioxide, Phys. Rev. B 84 (2011) 174105.

[23] S. T. Murphy, A. Chartier, L. Van Brutzel, J.-P. Crocombette, Free energy of xe incorporation at point defects and in nanovoids and bubbles in $\mathrm{UO}_{2}$, Phys. Rev. B 85 (2012) 144102.

[24] B. Basak, A. K. Sengupta, H. S. Kamath, Classical molecular dynamics simulations of $\mathrm{UO}_{2}$ to predict thermophysical properties, J. Alloys Compd. 360 (2003) 210-216.

[25] K. Govers, S. Lemehov, M. Hou, M. Verwerft, Comparison of interatomic potentials for $\mathrm{UO}_{2}$. Part I: Static calculations, J. Nucl. Mater. 366 (2007) 161.

[26] P. V. Nerikar, K. Rudman, T. G. Desai, D. Byler, C. Unal, K. J. McClellan, S. R. Phillpot, S. B. Sinnott, P. Peralta, B. P. Uberuaga, C. R. Stanek, Grain boundaries in uranium dioxide: Scanning electron microscopy experiments and atomistic simulations, J. Am. Ceram. Soc. 94 (6) (2011) 1893-1900.

[27] G. Henkelman, B. P. Uberuaga, H. Jonsson, A climbing image nudged elastic band method for finding saddle points and minimum energy paths, J. Chem. Phys. 113 (2000) 9901-9904.

[28] A. F. Voter, Introduction to the kinetic Monte Carlo method, in: K. E. Sickafus, E. A. Kotomin, B. P. Uberuaga (Eds.), Radiation Effects in Solids, Springer, NATO Publishing Unit, Dordrecht, The Netherlands, 2006.

[29] H. Blank, Properties of fission spikes in $\mathrm{UO}_{2}$ and $\mathrm{UC}$ due to electronic stopping power, Phys. Stat. Sol. A 10 (1972) 465478.

[30] T. Arima, K. Idemitsu, Y. Inagaki, Y. Tsujita, M. Kinoshita, E. Yakub, Evoluation of melting point of $\mathrm{UO}_{2}$ by molecular dynamics simulation, J. Nucl. Mater. 389 (2009) 149-154.

[31] M. Nomura, J. B. Adams, Self-diffusion along twist grain boundaries in Cu, J. Mater. Res 7 (1992) 3202-3212.

[32] M. Nomura, J. B. Adams, Interstitial diffusion along twist grain boundaries in Cu, J. Mater. Res 10 (1995) 2916-2924.

[33] A. Pedersen, H. Jónsson, Simulations of hydrogen diffusion at grain boundaries in aluminum, Acta Materialia 57 (2009) 4036.

[34] B. P. Uberuaga, L. J. Vernon, E. Martinez, A. F. Voter, The relationship between grain boundary structure, defect mobility, and grain boundary sink efficiency, Sci. Rep. 5 (2015) 9095.

[35] M. Sorensen, Y. Mishin, A. Voter, Diffusion mechanisms in $\mathrm{Cu}$ grain boundaries, Physical Review B 62 (6) (2000) 3658-3673.

[36] A. L.-S. Chua, N. A. Benedek, L. Chen, M. W. Finnis, , A. P. Sutton, A genetic algorithm for predicting the structures of interfaces in multicomponent systems, Nat. Mater. 9 (2010) 418422 . 


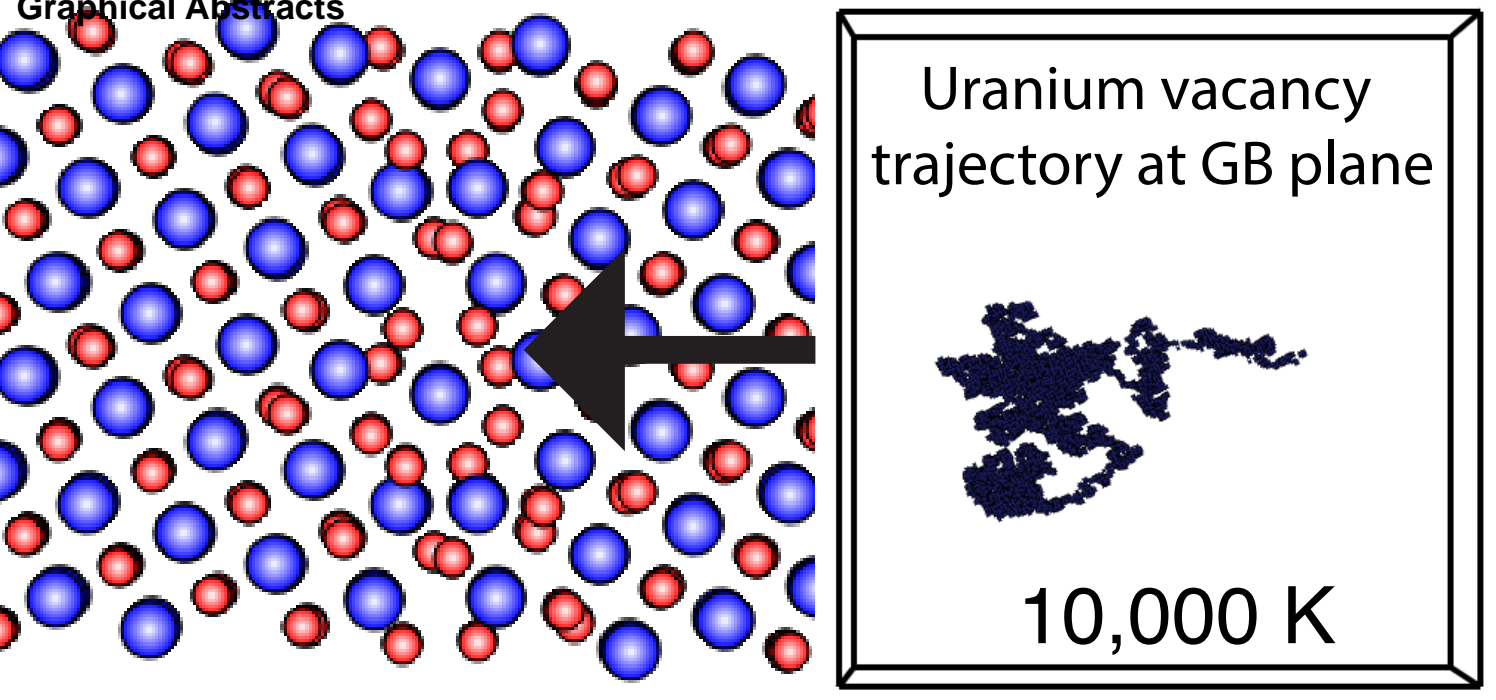

\section{Graphical Abstracts}

trajectory at GB plane

$10,000 \mathrm{~K}$ 